\title{
Optimizing the performance of metal-semiconductor-metal photodetectors by embedding nanoparticles in the absorption layer
}

\author{
Mahsa Naghipour \\ Sama Technical and Vocational College, Islamic Azad University, Miyandoab Branch, Miyandoab, Iran \\ Email address: \\ Mnaghipoor.elec@yahoo.com

\section{To cite this article:} \\ Mahsa Naghipour. Optimizing the Performance of Metal-Semiconductor-Metal Photodetectors by Embedding Nanoparticles in the \\ Absorption Layer. Journal of Electrical and Electronic Engineering. Special Issue: Research and Practices in Electrical and Electronic \\ Engineering in Developing Countries. Vol. 3, No. 2-1, 2015, pp. 78-82. doi: 10.11648/j.jeee.s.2015030201.27
}

\begin{abstract}
Metal-Semiconductor-Metal is one of the photodetectors that uses in optical detection. The characteristic of these detectors is improved by Plasmonic Science. Some of these characteristics include higher sensitivity, greater efficiency and improved optical absorption coefficient. Our predictions and the numerical results show high absorption in plasma-based combination of Metal-Semiconductor-Metal photodetectors in embedded Nano-grating and Metallic nanoparticles. The results of the simulation using FDTD simulation software shows that by optimizing the Nano grating metal parameters and embedded metallic nanoparticles, higher electric field is obtained, leading to higher absorption compared with the conventional MetalSemiconductor-Metal detector. This higher absorption is because of the higher ability of embedded metallic nanoparticles in absorbing the light and scattering properties by conduction electrons via stimulated resonance light from metallic nanoparticles.
\end{abstract}

Keywords: Absorption, Nanoparticles, Plasma

\section{Introduction}

Metal-Semiconductor-Metal is one of the photodetectors that uses in optical detection. The characteristic of these detectors is improved by Plasmonic Science. Some of these characteristics include higher sensitivity, greater efficiency and improved optical absorption coefficient. In these structures, the detection parameters were optimized by using metal diffraction mesh and nanoparticles. Also making the very small detectors becomes much easier by Plasmonic Science, which leads to the Optical Integrated circuits. Today, Plasmonic has wide applications in various fields of optical and electronic devices. Here some examples of the work done in the field of application of Plasmonic photodetectors will be discussed. In 2004, it was shown that by placing a Nano layer of light-absorbing semiconductor between two electrodes with a small gap, we can have a MSM detectector with more than $50 \%$ efficiency and cutoff of frequency of more than $300 \mathrm{GHz}$ [2]. In this structure, Nano semiconductors string was used between metal strings which have lower cross-section area than $\lambda / 8$. Results show that $75 \%$ efficiency and cutoff frequency of $500 \mathrm{GHz}$ can be obtained theoretically. This structure was built for $800 \mathrm{~nm}$ wavelength.

In 2006, the properties of plasmons surface was used to enhance the signal to noise ratio of an optical detector in the mid-infrared frequencies, by improving absorption [3].
This structure was designed for $9 / 8 \mu \mathrm{m}$ wavelength. The Results shows that with this structure, has attracted up to 250 -fold improvement in volume semiconductor material than conventional detectors on the same wavelength is obtained.

In 2007, an optimal design for MSM-PD with shoulder electrodes with sub-wavelength scale was provided where a single GaInNAs quantum wells, was used as the absorber layer, SP excitation at the metal-semiconductor boundary, improved field strength near the electrode and consequently increases the absorption in the QW.

In 2008, a circular diffraction grating structure was proposed to improve the signal to noise ratio of an infrared detector [1]. Simulations showed that the signal to noise ratio improve up to 2.5 times in this structure while in the linear diffraction grating, up to 1.7 times improvement can be obtained.

In 2010, MSM detector structure with a metallic diffraction grating was proposed [8]. SPP radiation emerging from the boundary of semiconductor and metallic diffraction grating, will lead to improve the absorption coefficient to the subwavelength aperture MSM up to 12 times due to a nonmetallic diffraction grating.

To further improve the absorption coefficient, a two-layer structure with MSM Plasmonic diffraction grating was proposed. The presented results show that the absorption 
coefficient of the MSM structure with sub wavelength apertures and diffraction grating, has improved 22 times.

Furthermore, in another study, it was suggested that instead of using a binary diffraction grating structure, the structure that uses the second diffraction grating layers of nanoparticles became operative [6]. The Plasmonic radiation in these NPs leads to improve the absorption coefficient. Showed that the absorption coefficient improved 28 times compared to conventional MSM and compared to MSM with metallic grating mesh and Sub wavelength aperture, 3.5 times improvement has gained. The nanoparticles that used in this article are silver and gold nanoparticles. Silver nanoparticles has better absorption coefficient.

\section{Plasma Nano Grating Metal}

Metal nano-grating in the MSM detector structures is used to act as micro-lenses that focus light into the gap below the wavelength limit and Resulting in substantial reduction of losses due to loss of light will return. Exact simulations and studies of surface plasmon Polarization(SPP) was published in the MSM PD structure, represents a very significant improvement in the transmission and absorption of light.

Recently optimized MSM PD structure show successful uptake in absorption. We propose a practical hybrid plasmonic MSM-PD structure incorporating plasmonic metal gratings in conjunction with localized surface plasmon resonance (LSPR) through embedded metal nanoparticles (NPs). Simulation results using Finite Difference Time Domain (FDTD) software demonstrate that this hybrid plasmonic MSM-PD structure yields enhancement in absorption compared to conventional non-plasmonic and plasmonic MSM-PD structures. This absorption enhancement is mainly due to the light-stimulated resonance induced by the conduction electrons of the embedded NPs.

\section{Plasmonic MSM PD Structure}

Figure 1 shows a typical MSM PD structure. Figure 2 shows the structure of a metal plasmonic nano-gratings which has used MSM PD and metal nanoparticles.

The absorption layer of plasma MSM PD can increases by using metallic nanoparticles embedded in the surface of the semiconductor. It is well known that the conduction electrons of such metal NPs induce light-stimulated resonance that enhances the optical absorption. This phenomenon is known as localized surface plasmon resonance (LSPR). However this LSPR is sensitive to the size, type, shape and refractive index of the metal NPs. Adopting the conventional dipole model, the absorption and scattering cross-sections of a nanoparticles, having a diameter much smaller than the wavelength, of the incident light, can be expressed as follows.

$$
\begin{gathered}
C_{a b s}=\frac{2 \pi}{\lambda} \operatorname{Im}(\alpha) \\
C_{s c a}=\frac{1}{6 \pi}\left(\frac{2 \pi}{\lambda}\right)^{4} \alpha^{2} \\
\alpha=3 V \frac{\varepsilon_{p}-\varepsilon_{s m}}{\varepsilon_{p}+\varepsilon_{s m}}
\end{gathered}
$$

$V$ : the volume of the particle $\varepsilon_{p}:$ dielectric function of the particles

$\varepsilon_{s m}$ : dielectric function of - surrounding medium

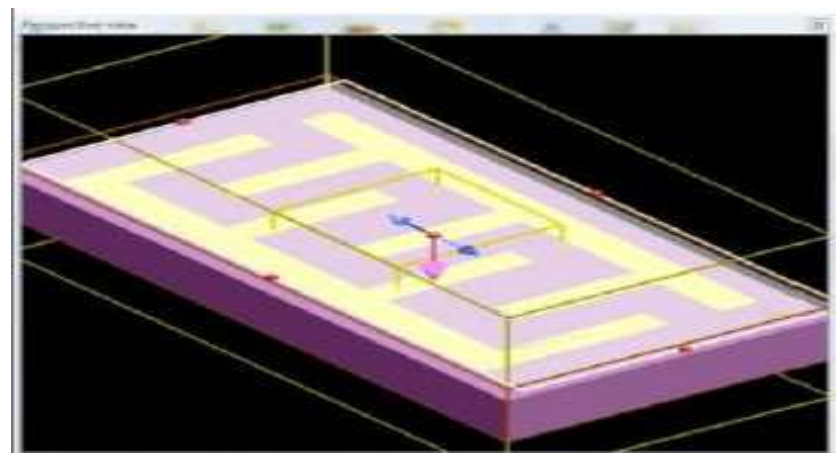

Figure 1. Conventional MSM PD Structure

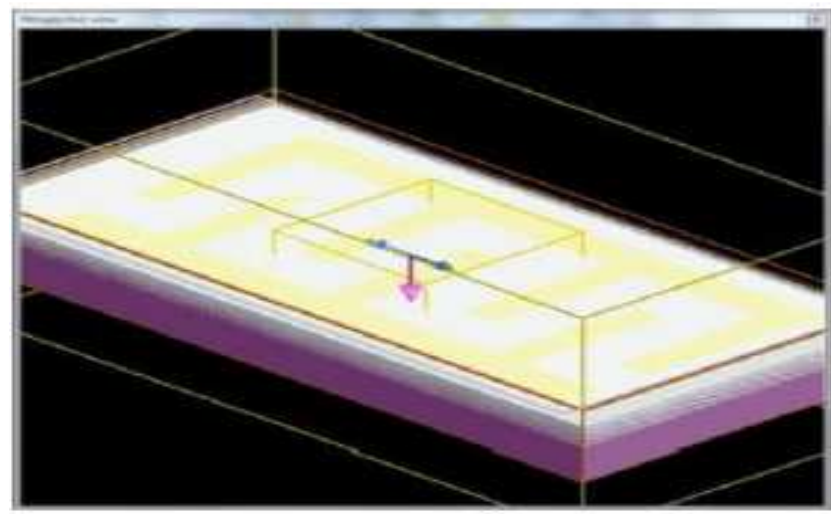

Figure 2. Optimized Plasmonic MSM PD Structure

\section{FDTD Simulation}

The 2D hybrid plasmonic MSM-PD structure was simulated by FDTD Software. In the design the refractive indices of $\mathrm{Au}$ and $\mathrm{Ag}, \varepsilon_{m}$, were obtained from the LorentzDrude model, while those of the GaAs and Ge, $\varepsilon_{\text {sub }}$, were assumed to be $3.66+6.28 \mathrm{e} 2 \mathrm{i}$ and $4.653+0.298 \mathrm{i}$, respectively.

Based on the simulation setup described above, parameters such as Substrate material, radius of nanoparticles, the nanoparticles and nano-metal grating and metal electrodes material were optimized for maximizing the absorption enhancement.

The absorption enhancement factor is defined as the ratio of the normalized power absorbed in the active area to the normalized power absorbed in a similar active area of a conventional MSM-PD structure without a metal nano grating.

\section{Results and Discussions}

E.1 : Embedded Metallic nanoparticles

Metallic nanoparticles embedded within a semiconductor increase the absorption of plasmon LSPR layer around the light. Selection of type, size, shape and type of host in nanoparticles material is critical in enhancing absorption of the LSPR.

E.1.1: Effect of host material in increasing the absorption

The simulated of the spectral shapes of the Plasmonic MSM PD with the gold and silver nanoparticles which host 
in $\mathrm{Si}, \mathrm{Sio} 2$ and $\mathrm{Ge}$ are shown in the below picture.

Figure 3, 4 and 5 shows the simulation result of the absorbed power in the host material with gold nanoparticles. The results are showed in the below figures.
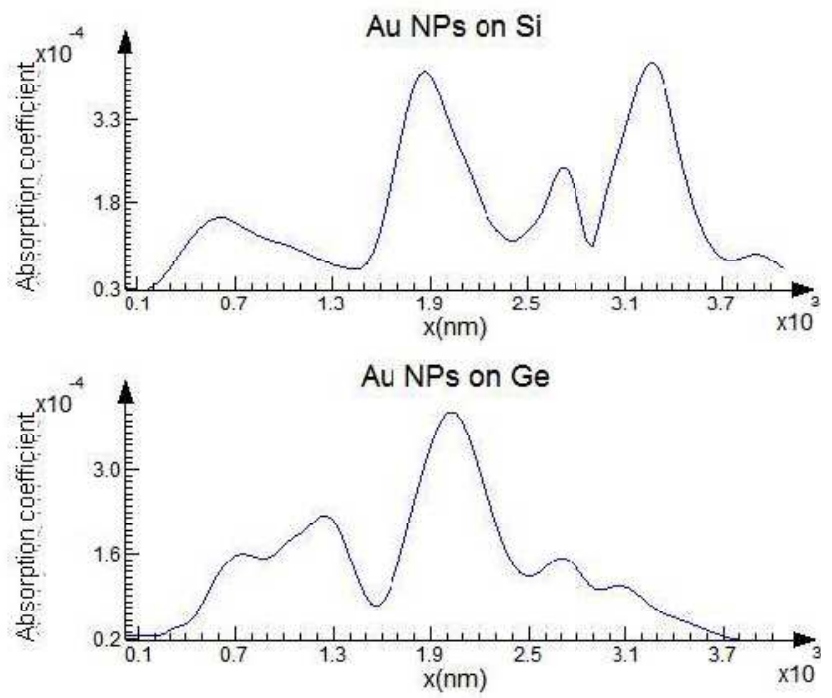

Figure 3,4. Increased absorption factors of gold nanoparticles on a host of Si and Ge

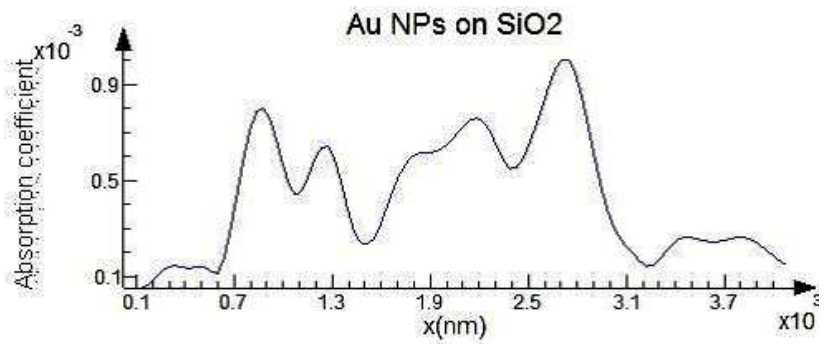

Figure 5. Enhancement absorption factor for gold nanoparticle on theSio2host

Figure 6,7 ,and 8 show simulation results of the power absorbed fractions for silver nanoparticles on the studied materials host. The results are as follows:


Figure 6, 7. Enhancement absorption factor for silver nanoparticles on theSi and Ge host



Figure 8. Enhancement absorption factor for silver nanoparticles on theSIO2 host

E.1.2: Effect of nanoparticle radius in the input photon absorption the effect of increasing the radius of the nanoparticle absorption is shown in the following figure.

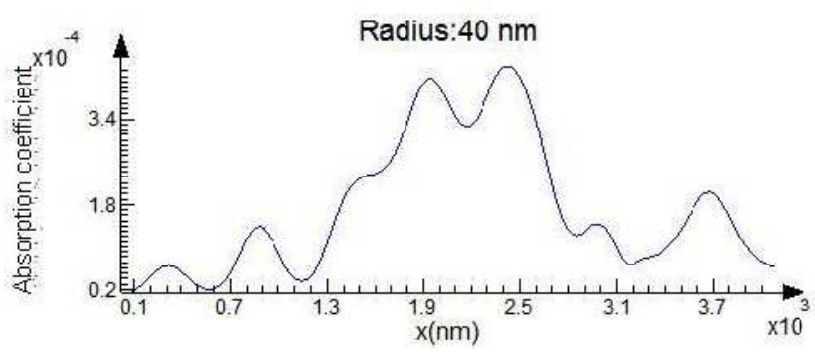

Figure 9. Absorption factor for the radius of - $40 \mathrm{~nm}$



Figure 10. Absorption factor for the radius of $-50 \mathrm{~nm}$

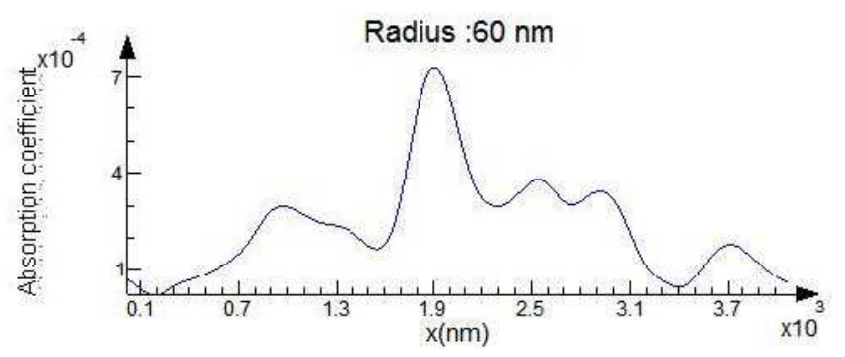

Figure 11. Absorption factor for the radius of $-60 \mathrm{~nm}$

Figures show that the radius of the nanoparticle plasmonic MSM PD structure has an important influence on increasing absorption. When the radius of metallic nanoparticles increases, the absorption coefficient also increases. The increase in the overall level of metallic nanoparticles is attributed to the interface on the host environment. It is important to note, however, that increasing the NP diameter also widens the absorption enhancement spectrum due to multimode light-stimulated resonance induced by the conduction electrons within the NPs. This spectral bandwidth broadening reaches a saturation point that leads to the appearance of two resonance peaks as shown in Figures.

E.2: Distribution of electric field

Figure 12 shows the simulation of electric field distribution MSM PD for conventional plasmonic and 
plasmonic MSM PD structure containing nanoparticles.

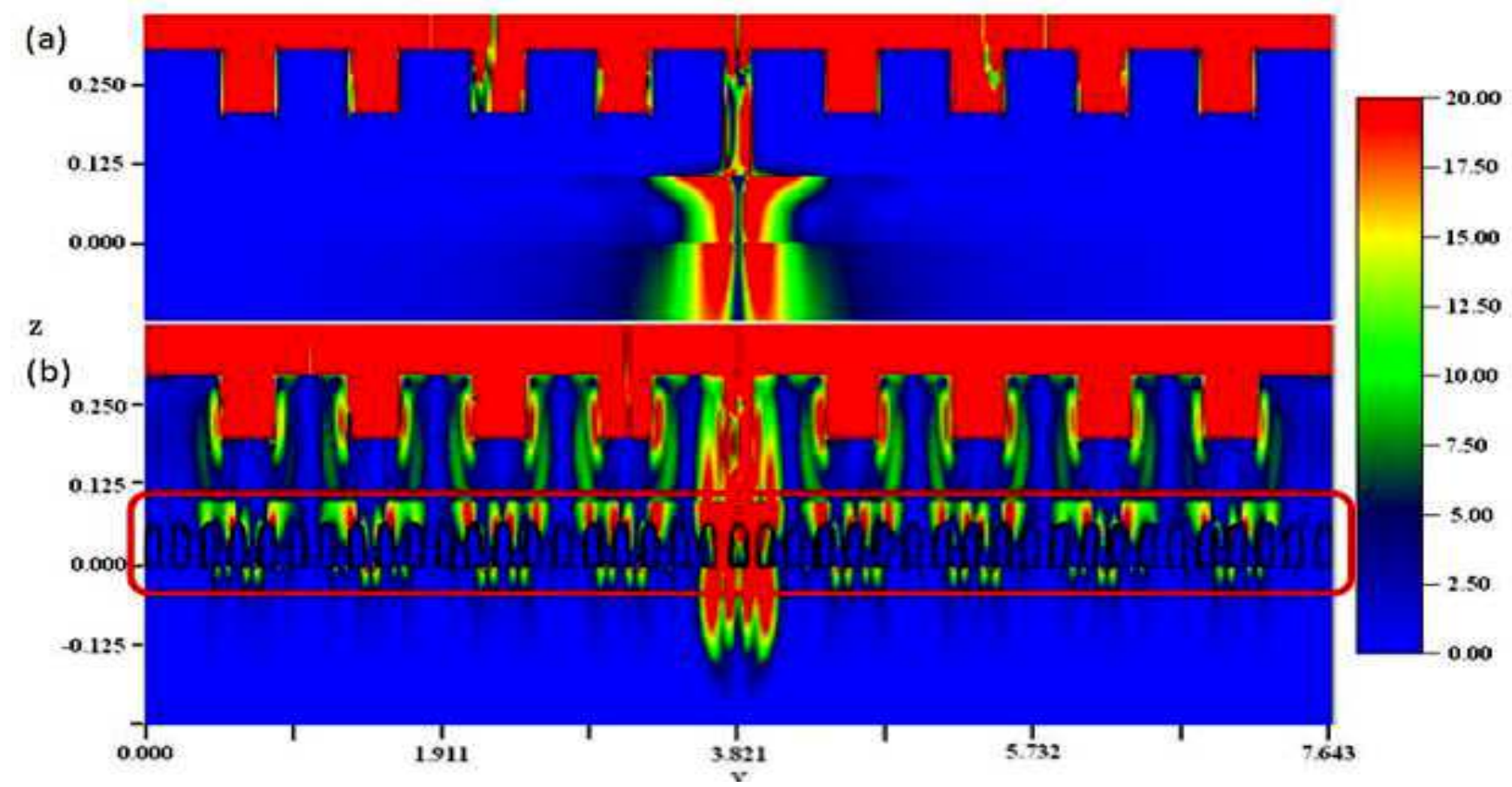

Figure 12. Simulated electric field distribution for MSM PD

It is important to note to that the SPP waveform in the grating metal and Influence of the electric field inside the GaAs in Plasmonic MSM PD structure. However the SPP wave is focused only on the outside of subwavelength gap and during the propagation, subwavelength gap exponentially decreases. Next figure reveals that the simulated resonant light with nanoparticles develop, the influence of the electric field level to plasmonic MSM PD. Absorption in the surface and the presence of nanoparticles and host, particularly increases because Scattering parameters of metallic nanoparticles, is tested over the diffusion path input light.

Such important parameter of a MSM light detector is the quantum efficiency and responsiveness of the detector. Quantum efficiency defines as the ratio of the generated number of electron-hole pairs to the number of incoming photons. The figure below shows the quantum efficiency for the optimized plasmonic structure of the detector using simulation software.

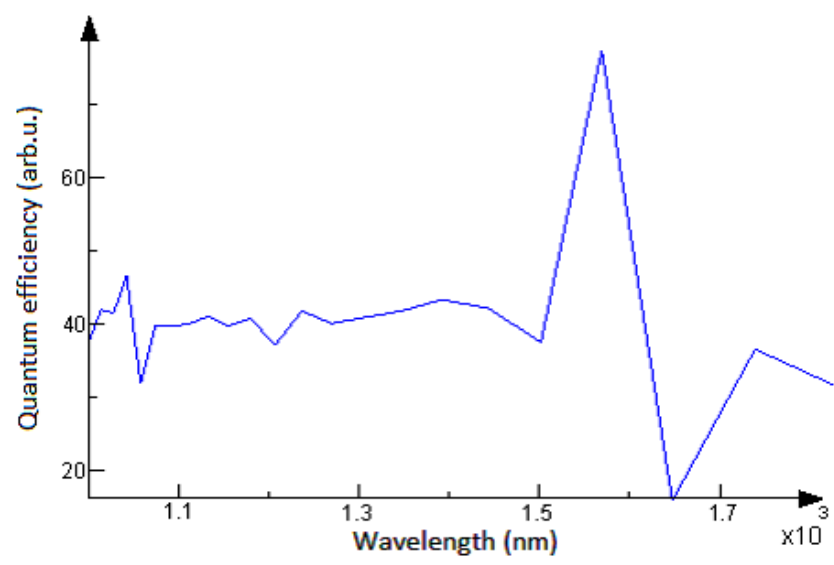

Figure 13. Quantum efficiency of the optimized plasmonic detector in the operating wavelength ( $1400-1600 \mathrm{~nm}$ ) by increasing wavelength, which leads to increased absorption of incoming photons and yields to increase in carrier production and in $1600 \mathrm{~nm}$ wavelength, efficiency is around $80 \%$.This represents a significant improvement in the efficiency of photon absorption.

Improvements of the Quantum efficiency may be a key factor in improving detector parameters.

The effects of the absorption wavelength of the semiconductor, and the effects of diffraction reduc the intensity of the absorption efficiency of light-sensitive semiconductor structures. Therefore, there is a tradeoff between speed and Quantum efficiency of conventional detectors.

Quantum efficiency of carriers generated by light emitted from the light of the other compound at best situation will be reduced as much as $10 \%$.

Achieving the Quantum efficiency of more than 50\% above the cutoff frequency of $300 \mathrm{GHz}$ need new solutions to Improve the absorption of nano-scale structures with a small capacitor.

Conventional MSM consists of the individual electrodes on the surface of the grown semiconductor absorber.

The high speed MSM PD structure will be produced by decreasing the distances between fingers but The Quantum efficiency significantly increases for Improvements of metal electrodes. Also the network acts as a high inverse mirror leading to efficient coupling of light entering the cavity and the absorption efficiency.

The structure of MSM PD in this article has analyzed and tried to improve by nanoparticles because it makes the absorption more efficient and produce an electron-hole.

As, already discussed, the Quantum efficiency increases 


\section{Discussion and Conclusion}

In this article, we tried to design and model a high speed metal-semiconductor-metal photo detector which contains nanoparticles for higher photon absorption.

For this purpose the structure of an electromagnetic wave radiated around improved plasmonic MSMPD has simulated by FDTD software. Wave transmission were studied showing different structures around which the structure has a higher absorption. In this simulation, the detector parameters such as the radius of the nanoparticle ( NPs-D ), nanoparticles and nano-gratings for embedding metal nanoparticles, and other parameters in order to increase the maximum absorption recovered.

Simulated electric field distribution for plasmonic and conventional MSMPD were also analyzed and concluded that simulated light resonant with nanoparticles develop the penetration of electric field While in the absence of nanoparticles, the electric field influence is less.

Simulation results show that the embedded nanoparticles increase light absorption and the metal nanoparticles, also silver nanoparticle is better than gold.

In addition to metallic nanoparticles, the host material is used for embedding gold and silver, were changed

Simulation results have shown that $\mathrm{Ge}$ and $\mathrm{Si}$ are suitable host materials for embedding Au and Ag NPs where localized surface plasmon resonance (LSPR) induced by the conduction electrons of the embedded metal NPs.

Between $\mathrm{Si}$ and $\mathrm{Ge}, \mathrm{Si}$ is one of the best material to achieve the high efficiency. This is resulted by the Absorption band simulation.

Improving the MSMPD plasmonic structure parameters shows, significantly increase in absorption compared with conventional MSMPD structure. This is simulated by the ability of the embedded Metallic nanoparticles in light absorption and scattering Properties around the resonant frequency which is done by the Conductivity of metallic nanoparticles electrons.

\section{Refrences}

[1] Bhat R. D. R,and, , N. C Panoiu., Brueck, S. R. J., and Osgood, R. M.,2008. "Enhancing the signal -to-noise ratio of an infrared photodetector with a circular metal grating,"Optics Express, vol.16, no.7, pp 4588-4596

[2] Collin, S, Pardo, F., Teissier, R., and Pelouard, J.L.2004 "Efficient light absorption in metal-semiconductor-metal nanostructures", Appl. Phys. Lett., vol.85(2).

[3] Collin, S., Pardo, F., Teissier, R., and Pelourad, J.2004 "Efficient light absorption in metal-semiconductor-metal nanostructures," Applied Physics Letters, vol. 85, no. 2, pp. 194-196.

[4] Das, N., Karar, A., Tan, C. L., Alameh, K., and Lee, Y. T.2011 "Impact of Nanograting Phase-Shift on Light Absorption Enhancement in Plasmonics-Based Metal-SemiconductorMetal Photodetectors," Advances in Optical Technologies, vol ) 504530)

[5] Hetterich, J., Bstian, Gippius, G., N. A., Tikhodeev, S. G., Plessen, G. von, and Lemmer, U.,.2007 "Optimized Design of Plasmonic MSM Photodetector," IEEE Journal of Quantum Electronics, vol. 43, no. 10, pp. 855-859.

[6] Tan, C. L., Karar, A., Alameh, K., and Lee, Y. T.,.2013 "Optical absorption enhancement of hybrid-plasmonic-based metal-semiconductor-metal photodetector incorporating metal nanogratings and embedded metal nanoparticles," Optics Express, vol. 21, no. 2, pp.1713-1725.

[7] Tan, C. L., Lysak, V. V., Das, N., Karaar, A., Alameh, K., Lee, Y. T., 2010. "Absorption enhancement of MSM Photodetector structure with a Plasmonic Double Geating Structure," 10th IEEE Conference in Nanotechnology (IEEE-NANO IEEE,), pp. $489-853$

[8] Tan, C. L., Lysk, V. V., Alameh, K., Lee, Y. T.,2010. "Absorption enhancement of $980 \mathrm{~nm}$ MSM photodetector with plasmonic grating structure," Optics Communications 283, 1763-1767. 